\title{
Dietary and non-dietary factors associated with the concentration of major gastrointestinal microbiota in healthy subjects
}

\author{
K. Whelan, A. Bartlett, A. Datta, C. R. H. Hedin, S. Kallis and E. Law \\ Nutritional Sciences Division, King's College London, London SE1 9NN, UK
}

Gastrointestinal (GI) microbiota are important in many aspects of clinical nutrition, including the management of inflammatory bowel disease, irritable bowel syndrome and enteral tube feeding diarrhoea ${ }^{(1)}$. However, the dietary and non-dietary factors (e.g. dietary fructooligosaccharide (FOS) intake, gender, age) associated with GI microbiota in healthy subjects, in the absence of these disease states, are less clear. In addition, whilst there is emerging data for a role of GI microbiota in murine models of overweight and obesity ${ }^{(2)}$, evidence in human subjects is sparse. Thus, the aim of the present study was to investigate the dietary and non-dietary factors associated with the major GI microbiota in healthy subjects.

Healthy subjects between the ages of 18 and 50 years were recruited to a cross-sectional survey of GI microbiota. Exclusion criteria were any diagnosed or suspected GI disorder, the use of antibiotics within the preceding 3 months, probiotics or prebiotics within the preceding month or the current use of any other drug or supplement known to impact on GI microbiota. Demographic data, weight and height were recorded and BMI calculated. Subjects then completed a $7 \mathrm{~d}$ semi-weighed food diary. Dietary FOS intake was calculated by converting dietary intake data into commodity intake data using the Food Commodity Intake Database (Department of Agriculture, Washington, DC, USA), which was then converted into FOS intake using commodity composition data ${ }^{(3)}$. A fresh faecal sample was collected, immediately processed and stored for analysis. The major GI microbiota were quantified using fluorescent in situ hybridisation using Cy3 labelled probes for total bacteria (EUB338), bifidobacteria (Bif164), Bacteroides-Prevotella (Bac303) and Eubacterium rectale-Clostridium coccoides (EREC482). Values were compared between groups using independent $t$ tests and correlations are presented as Pearson's correlation coefficients.

Forty-seven healthy subjects (fourteen males, thirty-three females) with a mean age of 31.4 (SD 7.8) years and mean BMI of 23.5 (SD 3.4) $\mathrm{kg} / \mathrm{m}^{2}$ were recruited. Mean FOS intake was 4.4 (SD 1.5) g/d; however, there was no significant correlation between dietary FOS intake and concentrations of faecal bifidobacteria $(r 0.29, P=0.845)$. Bifidobacteria concentrations were higher in females $(9.2$ (SD 0.7$)$ $\log _{10} / \mathrm{g}$ ) than males $\left(8.6(\mathrm{SD} 1.1) \log _{10} / \mathrm{g} ; P=0.012\right)$. There were significant negative correlations between age and total bacteria $(r-0.377$, $P=0.012)$, Bacteroides-Prevotella $(r-0.364, P=0.009)$ and E. rectale-C. coccoides $(r-0.325, P=0.026)$ but not between age and bifidobacteria $(r-0.029, P=0.848)$. There were no significant differences in any of the GI microbiota between those who were overweight or obese and those who were not, nor were there significant correlations between BMI and GI microbiota.

Faecal bifidobacteria were higher in females, despite them having lower FOS intakes. Despite extensive evidence of the bifidogenic effect of supplemental FOS, the current study failed to demonstrate an association between dietary FOS intake and faecal bifidobacteria. Many of the GI microbiota declined significantly with age, an observation previously only reported in those $>60$ years of age. Despite emerging evidence of a role for the microbiota in overweight and obesity, the study failed to find any differences in concentrations, although microbiota profile rather than only concentrations are likely to be of importance. Research relating to the associations between dietary and non-dietary factors and the GI microbiota in diseases of relevance to clinical nutrition is warranted.

1. Hart AL, Stagg AJ, Frame M et al. (2002) Aliment Pharmacol Ther 16, 1383-1393.

2. Turnbaugh PJ, Ley RE, Mahowald MA et al. (2006) Nature 444, 1027-1031.

3. Van Loo JAE, Coussement P, Leenher LD et al. (1995) Crit Rev Food Sci Nutr 35, 525-552. 